\title{
Article
}

\section{Interventions for the management of abdominal pain in ulcerative colitis}

Iheozor-Ejiofor, Zipporah, Gordon, Morris and Akobeng, Anthony K Available at http://clok.uclan.ac.uk/33435/

Iheozor-Ejiofor, Zipporah, Gordon, Morris ORCID: 0000-0002-1216-5158 and Akobeng, Anthony K (2020) Interventions for the management of abdominal pain in ulcerative colitis. Cochrane Database of Systematic Reviews, 4 .

It is advisable to refer to the publisher's version if you intend to cite from the work. http://dx.doi.org/10.1002/14651858.cd013589

For more information about UCLan's research in this area go to

http://www.uclan.ac.uk/researchgroups/ and search for < name of research Group>.

For information about Research generally at UCLan please go to http://www.uclan.ac.uk/research/

All outputs in CLoK are protected by Intellectual Property Rights law, including Copyright law. Copyright, IPR and Moral Rights for the works on this site are retained by the individual authors and/or other copyright owners. Terms and conditions for use of this material are defined in the policies page.

\section{CLoK}

Central Lancashire online Knowledge www.clok.uclan.ac.uk

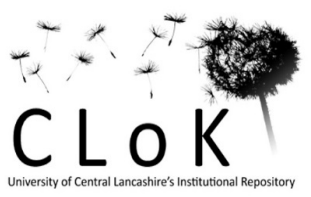




\section{Cochrane}

Library

Cochrane Database of Systematic Reviews

\section{Interventions for the management of abdominal pain in ulcerative colitis (Protocol)}

Iheozor-Ejiofor Z, Gordon M, Akobeng AK

Iheozor-Ejiofor Z, Gordon M, Akobeng AK.

Interventions for the management of abdominal pain in ulcerative colitis (Protocol).

Cochrane Database of Systematic Reviews 2020, Issue 4. Art. No.: CD013589.

DOI: 10.1002/14651858.CD013589.

www.cochranelibrary.com

Interventions for the management of abdominal pain in ulcerative colitis (Protocol) 
TABLE OF CONTENTS

HEADER 1

ABSTRACT

BACKGROUND

OBJECTIVES

METHODS

ACKNOWLEDGEMENTS

REFERENCES

APPENDICES

HISTORY

CONTRIBUTIONS OF AUTHORS

DECLARATIONS OF INTEREST 
[Intervention Protocol]

\section{Interventions for the management of abdominal pain in ulcerative colitis}

Zipporah Iheozor-Ejiofor ${ }^{1}$, Morris Gordon ${ }^{1}$, Anthony K Akobeng ${ }^{2}$

1School of Medicine, University of Central Lancashire, Preston, UK. ${ }^{2}$ Sidra Medicine, Doha, Qatar

Contact address: Anthony K Akobeng, aakobeng@sidra.org, akobeng@aol.com.

Editorial group: Cochrane IBD Group.

Publication status and date: New, published in Issue 4, 2020.

Citation: Iheozor-Ejiofor Z, Gordon M, Akobeng AK. Interventions for the management of abdominal pain in ulcerative colitis (Protocol). Cochrane Database of Systematic Reviews 2020, Issue 4. Art. No.: CD013589. DOI: 10.1002/14651858.CD013589.

Copyright @ 2020 The Cochrane Collaboration. Published by John Wiley \& Sons, Ltd.

\section{A B S T R A C T}

\section{Objectives}

This is a protocol for a Cochrane Review (intervention). The objectives are as follows:

To assess the efficacy and safety of interventions for managing abdominal pain in ulcerative colitis. 


\section{B A C K G R O U N D}

\section{Description of the condition}

Ulcerative colitis (UC) is a chronic inflammation of the colon characterised by periods of relapse and remission (Ordas 2012). It starts in the rectum and extends throughout the colon. UC and Crohn's disease (CD) are the two most common inflammatory bowel diseases (IBD). However, UC tends to be more common than $C D$, with an estimated prevalence of 90 to 505 cases per 100,000 people in North American and northern Europe (Conrad 2014). Whilst prevalence has been historically higher in Western countries, its incidence in industrialised parts of Asia and Latin America is on the rise. The cause of UC is not known but is believed to be associated with certain genetic and environmental factors. There is a higher risk in Ashkenazi Jews, people with a family history of the disease and those who live in Western countries (da Silva 2014).

Some of the symptoms of active UC include abdominal pain, bloody stools and diarrhoea. These symptoms can be managed using medical interventions such as 5-aminosalicylates, oral corticosteroids, azathioprine or mecarptopurine (Iskandar 2015; Iheozor-Ejiofor 2019) and by surgery in around 20 to $30 \%$ of sufferers who do not successfully attain remission with drugs (Ordas 2012). However, studies have shown that abdominal pain persists in up to one third of UC patients in remission (Coates 2013). This has been attributed to the coexistence of functional bowel disorders such as IBS (irritable bowel syndrome). It is postulated that as the symptoms of IBS and IBD share common underlying psychological (for example anxiety and depression) and clinical factors (for example colonic inflammation), an overlap of these factors may trigger a myriad of events which result in persistent pain in sufferers (Deberry 2014).

\section{Description of the intervention}

\section{Pharmacological interventions}

IBD medication can reduce inflammation and associated pain by inducing remission. Where pain persists in the absence of inflammation, it can be managed with pain-relieving medication such as antispasmodics, non-steroidal anti-inflammatory drugs (NSAID), cyclooxygenate- 2 inhibitors (COX-2) and narcotics (Srinath 2012). Short-term use is advised due to the potential adverse effects of some of these drugs,

Antispasmodics are a heterogenous group of drugs which can relax intestinal muscles. Hyoscyamine and dicycloamine are the most common antispasmodics that are used in IBD. NSAIDs are a group of non-chemically related compounds which have analgesic effects. They reduce inflammation by inhibiting the production of prostaglandins (Cavkaytar 2019). Examples include ibuprofen, sulphasalazine, indomethacin and paracetamol. Some of these drugs are available as over-the counter drugs. Narcotics are psychoactive compounds with sleep-inducing properties such as opiates and opioids, morphine, codeine, etc. Even though narcotics have historically been viewed in a negative light, observational studies indicate that they are commonly used not only for adults but also children with IBD (Buckley 2013; Buckley 2015). Finally, neuromodulators have been used in functional abdominal pain syndromes and as such for abdominal pain in inflammatory bowel disease (Mikocka-Walus 2020).

\section{Non-pharmacological interventions}

Non-pharmacological interventions used in managing abdominal pain may include psychological interventions, lifestyle advice, dietary interventions and alternative medicine. These interventions are generally considered less invasive and may be used as adjuvant treatment.

Psychological therapies are based on theories of human behaviour. Cognitive behavioural therapy, stress management, and coping skills training are the most common psychological interventions used. These are an interesting set of therapies as the specific interventions delivered can be very heterogenous and as such it is key to consider the specific evidence and conceptual alignment of the approach delivered to understand 'what' the therapy was, as well as 'whether' it is effective.

Dietary factors include alcohol elimination and the use of supplements with prebiotic properties. Dietary factors have been considered, with some evidence of impact (Norton 2017). There is also interest in the use of probiotics for functional abdominal pain syndromes, given their impact on the gut microbiome and the reduction in inflammatory processes they may produce (IheozorEjiofor 2019).

Alternative medicine such as acupuncture and TENS, which have been used with other conditions such as IBS, are being more frequently used in IBD patients albeit with limited evidence (Srinath 2012). Acupuncture is a complementary therapy which is generally used for pain unresponsive to standard therapy (Wilkinson 2007). There are various techniques used in acupuncture such as basic needling, laser acupuncture, and electroacupuncture.

\section{How the intervention might work}

The cause of the abdominal pain could require a targeted approach.

\section{Pharmacological interventions}

Antispasmodics often have mixed mechanisms of action, but generally they tend to suppress intestinal spasms resulting from inflammation or obstruction (Srinath 2012). Pharmacological interventions may have associated adverse effects. For example, it is widely known that NSAIDs may increase the risk of disease flare-up or exacerbation in IBD patients (Klein 2010). In addition to offering short-term relief, there seem to be concerns among IBD sufferers about the stigma of addiction associated with the use of opioids.The use of psychoactive drugs can also lead to heavy dependence on them and a higher risk of mortality (Coates 2013). In IBD patients, tapering off narcotics could trigger withdrawal symptoms which mimic IBD symptoms (Pauly 2017), thus complicating further treatment. Therefore, long-term use for IBD pain relief is not recommended.

\section{Non-pharmacological interventions}

Pain resulting from strictures can be eliminated by the introduction of foods which can pass through with ease thereby preventing intestinal pain (Srinath 2012). It has been postulated that recurrent pain tends to lead to coping behaviours which worsen the experience of pain. Psychological techniques such as cognitive behavioural therapy work by targeting and stopping these negative coping mechanisms that affect how people deal with pain (Norton 2017). The mechanism of action of alternative and complementary 
therapies in itself is highly complex, but they are common use in wider society and in turn are used by sufferers of UC.

\section{Why it is important to do this review}

Abdominal pain is a major driver for the use of healthcare facilities in IBD sufferers. For about $70 \%$ of people with IBD, it is the main reason for seeking medical attention. This puts a financial strain on healthcare systems amounting to billions every year (Ghosh 2015). For the patient, it can lead to psychological problems, loss of earnings and a general decline in quality of life. Therefore, effective pain management is vital. Pain management has been highlighted as a priority topic for research by IBD patient groups and charities but is currently not covered in the National Institute for Health and Care Excellence (NICE) or European Crohn's and Colitis Organisation (ECCO) guidelines (NICE 2019; ECCO 2010). Whilst several non-Cochrane systematic reviews have assessed interventions for pain management in IBD, there is currently none which has assessed the efficacy and safety of these interventions specifically in UC. Even though this review covers interventions that have been previously assessed in previously published Cochrane systematic reviews (Iheozor-Ejiofor 2019; Limetkai 2019; Timmer 2011; Kafil 2018), the focus is only on studies that have been conducted for the purpose of providing relief for abdominal pain.

\section{O B J E C T IVES}

To assess the efficacy and safety of interventions for managing abdominal pain in ulcerative colitis.

\section{METHODS}

\section{Criteria for considering studies for this review}

\section{Types of studies}

All published, unpublished and ongoing randomised trials that compare interventions for the management of abdominal pain with other active interventions or standard therapy, placebo or no therapy. We will exclude studies that do not report on any abdominal pain outcomes.

Induction or maintenance studies for UC that impact abdominal pain as a proxy of disease state will not be considered. Similarly, studies considering other pain in IBD, such as pain associated with extra-manifestations will not be considered.

Studies including patients with both $U C$ and $C D$ will only be included if the data for UC patients can be disaggregated.

\section{Types of participants}

People with UC who are experiencing abdominal pain.

\section{Types of interventions}

- Pharmacological treatments (e.g. antispasmodics, antidepressants, laxatives, antidiarrhoeal agents, antibiotics, analgesics, anti-reflux agents, antiemetic agents, antimigraine agents, antihistaminic agents, serotonergic agents and psychoactive drugs)

- Behaviour therapy (e.g. cognitive-behavioral therapy, hypnotherapy)

- Lifestyle advice (e.g. advice on physical activity including exercise)
- Dietary interventions (e.g. FODMAP, additional fibre intake, decrease in gas producing foods, extra fluid intake, lactulose- / gluten- / histamine-free diet)

- Prebiotics and probiotics

- Alternative medicines (e.g. acupuncture, homeopathy, bodyoriented therapy, musculoskeletal therapy (osteopathy/ chiropractic), yoga)

\section{Types of outcome measures}

\section{Primary outcomes}

- Treatment success as defined by the author

- Abdominal pain frequency or change in frequency of pain

- Abdominal pain intensity or change in pain intensity using any validated scale

- Withdrawal due adverse events

\section{Secondary outcomes}

- Anxiety/depression

- Quality of life, using validated measures.

- Adverse events

- Serious adverse events

\section{Timing of outcome measurement}

Where outcomes are measured at multiple time points, they will be subgrouped into three time periods: short-term (within 1 month of the intervention), medium-term ( $<1$ month to 6 months) and longterm (more than 6 months).

\section{Search methods for identification of studies}

\section{Electronic searches}

We will search the following sources from the inception of each database to the date of search and will place no restrictions on the language of publication:

- Cochrane Central Register of Controlled Trials (CENTRAL) via the Cochrane Register of Studies Online (CRSO)

- MEDLINE (Ovid MEDLINE ALL from 1946)

- PsycINFO via Ovid

- CINAHL via EBSCO (Cumulative Index to Nursing and Allied Health Literature)

- AMED (Allied and Complementary Medicine database) via Ovid

- ClinicalTrials.gov (www.clinicaltrials.gov)

- World Health Organization International Clinical Trials Registry Platform (ICTRP) (www.who.int/trialsearch/)

For detailed search strategies, see Appendix 1.

\section{Searching other resources}

As complementary search methods, we will carefully check relevant systematic reviews for studies for potential inclusion in our review. In addition, we will scrutinize the references of included studies in our review. We will seek unpublished trials by contacting experts in the field and we will scan the Internet and abstracts submitted to major international congresses from the three years prior to the search to capture any studies presented but not yet published in full. 
We will attempt to obtain translations of papers when necessary.

\section{Data collection and analysis}

We will carry out data collection and analysis according to the methods recommended in the Cochrane Handbook for Systematic Reviews of Interventions (Higgins 2011).

\section{Selection of studies}

Two review authors will independently screen the titles and abstracts identified from the literature search. We will discard studies which do not meet the inclusion criteria. We will then obtain the full report of studies which appear to meet our inclusion criteria or for which there is insufficient information to make a final decision. When these articles are obtained, two review authors will independently assess them to establish whether the studies meet the inclusion criteria. We will resolve disagreements by discussion, with a third review author consulted if resolution is not possible. We will enter studies rejected at this or subsequent stages in the 'Characteristics of excluded studies' tables and record the main reason for exclusion.

\section{Data extraction and management}

Two authors will carry out data extraction independently using piloted data extraction forms. We will extract relevant data from full-text articles that meet the inclusion criteria. If reported, information will be collected on:

- Trial setting: country and number of trial centres

- Methods: study design, total study duration and date

- Participant characteristics: age, socio-demographics, ethnicity, diagnostic criteria and total number

- Eligibility criteria: inclusion and exclusion criteria

- Intervention and comparator

- Outcomes: outcome definition, unit of measurement and time of collection

- Results: number of participants allocated to each group, missing participants, sample size

- Funding source

\section{Assessment of risk of bias in included studies}

During data extraction, two review authors will independently assess all studies meeting the inclusion criteria for their risk of bias using criteria outlined in the Cochrane Handbook for Systematic Reviews of Interventions (Higgins 2011). The domains that will be assessed are as follows:

- Sequence generation (selection bias)

- Allocation concealment (selection bias)

- Blinding of participants and personnel (performance bias)

- Blinding of outcome assessment (detection bias)

- Incomplete outcome data (attrition bias)

- Selective reporting (reporting bias)

- Other bias

We will judge the studies to be at either low, high or unclear risk of bias for each domain assessed, based on the guidance in Higgins 2011.
After data extraction, the two review authors will compare the extracted data to discuss and resolve discrepancies before the data are transferred into the Characteristics of included studies table. For cluster RCTs, we intend to judge risk of bias as prescribed in Section 16.3.2 of the Cochrane Handbook.

\section{Measures of treatment effect}

For the dichotomous outcomes, we will express treatment effect as risk ratios (RR) with corresponding $95 \% \mathrm{Cl}$. For continuous outcomes, we will express the treatment effect as mean difference (MD) with $95 \% \mathrm{Cl}$. However, if the studies assess the same continuous outcome in different ways, we will estimate the treatment effect using the standardised mean difference (SMD). We will present SMDs as standard deviation units and interpret as follows: 0.2 represents a small elect, 0.5 a moderate elect and 0.8 a large elect (Cohen 1988).

\section{Unit of analysis issues}

The participant will be the unit of analysis. For studies comparing more than two intervention groups, we will make multiple pairwise comparisons between all possible pairs of intervention groups. To avoid double counts, we intend to divide out shared intervention groups evenly among the comparisons. For dichotomous outcomes, we plan to divide up both the number of events and the total number of patients. For continuous outcomes, we will only divide up the total number of participants and leave the means and standard deviations unchanged. Cross-over studies will only be included if data are separately reported before and aRer cross-over. We do not anticipate any cluster RCTs, however, study data will only be used if the authors have used appropriate statistical methods in taking clustering elect into account. We will also exclude cluster RCTs in a sensitivity analysis to assess their impact on the results.

\section{Dealing with missing data}

We aim to contact authors where there is missing data or studies have not reported data in sufficient detail. We will attempt to estimate missing standard deviations using relevant statistical tools and calculators if studies report standard errors. Studies which fail to report measures of variance will be judged as being at high risk of selective reporting bias.

\section{Assessment of heterogeneity}

We plan to scrutinise studies to ensure that they are clinically homogenous in terms of participants, intervention, comparator and outcome. To test for statistical heterogeneity, we will use a chi ${ }^{2}$ test. A P-value of less than 0.1 will give an indication of the presence of heterogeneity. Inconsistency will be quantified and represented by the ${ }^{2}$ statistic. The thresholds are interpreted as follows (Higgins 2011):

$0 \%$ to $40 \%$ : might not be important

$30 \%$ to $60 \%$ : may represent moderate heterogeneity

$50 \%$ to $90 \%$; may represent substantial heterogeneity

$75 \%$ to $100 \%$ : considerable heterogeneity 


\section{Assessment of reporting biases}

Most reporting biases will be minimised by using an inclusive search strategy. We will investigate publication bias using a funnel plot if there are 10 or more studies. The magnitude of publication bias will be determined by visual inspection of the asymmetry of the funnel plot. In addition, we will test funnel plot asymmetry by performing a linear regression of intervention effect estimate against its standard error, weighted by the inverse of the variance of the intervention effect estimate (Egger 1997).

\section{Data synthesis}

To summarise the study characteristics, we intend to conduct a narrative synthesis of all the included studies. We will then carry out a meta-analysis if there are two more studies that have assessed similar populations, interventions and outcomes. Studies from paediatric population, adult population and dilerent subintervention types will be analysed separately. We will use Review Manager 5.3 (RevMan 2014). Study data will be synthesised using the random-effects model. We will combine effect estimates of studies which report data in a similar way, in the metaanalysis. We will pool RRs for dichotomous outcomes and MDs for continuous outcomes alongside $95 \%$ confidence intervals. Where we are unable to carry out a meta-analysis (e.g. due to lack of uniformity in data reporting), we will present a narrative summary of the included studies.

\section{Subgroup analysis and investigation of heterogeneity}

If heterogeneity is detected, we will investigate possible causes and address them using methods described in Higgins 2011. We will also undertake subgroup analyses of potential effect modifiers if there are 10 studies or more. We have identified several potential modifiers of effect:

- Disease activity (active versus inactive disease)

- Pain location

- Disease location

It is recognised that the nature of the studies likely to be included in this review may be capricious and heterogenous in a number of key clinical and methodological ways that cannot be fully predicted. If such factors are identified and become relevant to ensure integrity of the analysis, modifications to this list may be needed. These will be fully reported by the authors.

\section{Sensitivity analysis}

Where possible, we plan to undertake a sensitivity analysis on the primary outcome of 'treatment success', to assess whether the findings of the review are robust to the decisions made during the review process. In particular, we intend to exclude studies at high or unclear risk of bias due to allocation bias and performance bias, from analyses that have a mix of studies with dilerent risk of bias judgements. Where data analyses include studies with reported and estimated SDs, plan to exclude those with estimated standard deviations to assess whether this alects the findings of the review. We will investigate whether the choice of model (fixed versus random) impacts the results to explore heterogeneity.

\section{Summary of findings and assessment of the certainty of the evidence}

We will present the main results in a 'Summary of findings' table. Each comparison and primary outcome will be exported to GRADEprofiler software (developed by the Grades of Recommendations, Assessment, Development and Evaluation (GRADE) Working Group) for quality assessment (GRADE 2015). Data permitting, we intend to present five summary of findings tables in the following hierarchy: comparisons involving pain-relieving drugs, behavioural therapy, lifestyle advice, dietary interventions, prebiotics and probiotics. We will include all four primary outcomes. We will apply GRADE to any additional comparisons and present these in additional tables. Based on risk of bias, inconsistency, imprecision, indirectness and publication bias, we will rate the quality of the evidence for each outcome as high, moderate, low or very low. These ratings have been defined as follows:

- High: further research is very unlikely to change our confidence in the estimate of effect

- Moderate: further research is likely to have an important impact on our confidence in the estimate of effect and may change the estimate

- Low: further research is very likely to have an important impact on our confidence in the estimate of effect and is likely to change the estimate

- Very low: any estimate of effect is very uncertain

We will justify all decisions to downgrade the quality of studies using footnotes and we will make comments to aid reader's understanding of the review where necessary.

\section{ACKNOWLEDGEMENTS}

Funding for ZIE and partial funding for MG was provided through a larger NIHR Cochrane Programme Grant in the UK.

Maria-Inti Metzendorf (Information Specialist of the Cochrane Metabolic and Endocrine Disorders Group) developed the original search strategies, which were run by Yuhong Yuan (Information Specialist, Cochrane Upper GI and Pancreatic Diseases Group).

We thank the following peer reviewers for their time and comments: John Marshall (Department of Medicine, Division of Gastroenterology

McMaster University, Canada), Laurie Keefer (Division of Gastroenterology, School of Medicine at Mount Sinai, New York, USA) and Rachel Richardson (Cochrane Abdomen and Endocrine Network) 


\section{REFERE N C E S}

\section{Additional references}

\section{Buckley 2013}

Buckley JP, Kappelman MD, Allen JK, Van Meter SA, Cook SF. The burden of comedication among patients with inflammatory bowel disease. Inflammatory Bowel Disease 2013; 19(13):2725-36. [DOI: 10.1097/01.MIB.0000435442.07237.a4]

\section{Buckley 2015}

Buckley JP, Cook SF, Allen JK, and Kappelman MD. Prevalence of Chronic Narcotic Use Among Children With Inflammatory Bowel Disease. Clinical Gastroenterology and Hepatology 2015; 13(2):310-5. [DOI: 10.1016/j.cgh.2014.07.057]

\section{Cavkaytar 2019}

Cavkaytar O, du Toit G, and Caimmi D. Characteristics of NSAIDinduced hypersensitivity reactions in childhood. Pediatric Allergy and Immunology 2019; 30(1):25-35. [DOI: 10.1111/ pai.12980]

\section{Coates 2013}

Coates MD, Lahoti M, Binion DG, Szigethy EM, Regueiro $\mathrm{MD}$ and Bielefeldt K. Abdominal Pain in Ulcerative Colitis. Inflammatory Bowel Diseases. Inflammatory Bowel Diseases 2013; 19(10):2207-14. [DOI: 10.1097/MIB.0b013e31829614c6 \%J Inflammatory Bowel Diseases]

\section{Conrad 2014}

Conrad K, Roggenbuck D, and Laass MW. Diagnosis and classification of ulcerative colitis. Autoimmunity Reviews 2014; 13(4):463-6. [DOI: https://doi.org/10.1016/j.autrev.2014.01.028]

\section{da Silva 2014}

da Silva BC, Lyra AC, Rocha R, and Santana GO. Epidemiology, demographic characteristics and prognostic predictors of ulcerative colitis. World journal of gastroenterology 2014; 20(28):9458-67. [DOI: 10.3748/wjg.v20.i28.9458]

\section{Deberry 2014}

Deberry JJ, Bielefeldt K, Davis BM, Szigethy EM, Hartman DJ, and Coates MD. Abdominal Pain and the Neurotrophic System in Ulcerative Colitis. Inflammatory Bowel Diseases 2014; 20(12):2330-9. [DOI: 10.1097/MIB.0000000000000207\%J Inflammatory Bowel Diseases]

\section{ECCO 2010}

Dignass A, Van Assche G, Lindsay JO, Lemman M, Söderholm J, The second European evidence-based Consensus on the diagnosis and management of Crohn's disease: Current management. Journal of Crohn's and Colitis 2010; 4(1):28-62. [DOI: 10.1016/j.crohns.2009.12.002]

\section{Egger 1997}

Egger M, Smith GD, Minder C. Bias in meta-analysis detected by a simple, graphical test. BMJ 1997; 315(7109):629-34. [DOI: 10.1136/bmj.315.7109.629]

\section{Ghosh 2015}

Ghosh N, . A UK cost of care model for inflammatory bowel disease. Frontline Gastroenterol 2015; 6:169-74.

\section{GRADE 2015 [Computer program]}

McMaster University GRADEpro GDT: GRADEpro Guideline Development Tool. Available from: www.gradepro.org: McMaster University, 2015 (developed by Evidence Prime, Inc).

\section{Greenley 2013}

Greenley RN, Kunz JH, Schurman JV, Swanson E. Abdominal Pain and Health Related Quality of Life in Pediatric Inflammatory Bowel Disease. Journal of Pediatric Psychology 2013; 38(1):63-71. [DOI: 10.1093/jpepsy/jss097]

\section{Higgins 2011}

Higgins JPT, . Cochrane Handbook for Systematic Reviews of Interventions. Available from www.handbook.cochrane.org. Version 5.1.0 [updated March 2011] edition. The Cochrane Collaboration, 2011.

\section{Iheozor-Ejiofor 2019}

Iheozor-Ejiofor Z, Gordon M, Clegg A, Freeman SC, GjuladinHellon $\mathrm{T}$, . Interventions for maintenance of surgically induced remission in Crohn's disease: a network meta-analysis. Cochrane Database of Systematic Reviews 2019, Issue 9. [DOI: 10.1002/14651858.CD013210.pub2]

\section{Iskandar 2015}

Iskandar HN, Dhere T, Farraye FA. Ulcerative colitis: update on medical management. Current Gastroenterology Reports 2015; 17(11):44. [DOI: https://doi.org/10.1007/s11894-015-0466-9]

\section{Kafil 2018}

Kafil TS, Nguyen TM, MacDonald JK, Chande N. Cannabis for the treatment of Crohn's disease. Cochrane Database of Systematic Reviews 2018, Issue 11. [DOI: 10.1002/14651858.CD012853]

\section{Klein 2010}

Klein A and Eliakim R. Non Steroidal Anti-Inflammatory Drugs and Inflammatory Bowel Disease. Pharmaceuticals (Basel) 2010; 3(4):1084-92. [DOI: 10.3390/ph3041084]

\section{Limetkai 2019}

, , , , , , , et al. Dietary interventions for induction and maintenance of remission in inflammatory bowel disease. Cochrane Database of Systematic Reviews 2019, Issue 2. [DOI: 10.1002/14651858.CD012839.pub2]

\section{Mikocka-Walus 2020}

, , , , . Antidepressants in inflammatory bowel disease. Nat Rev Gastroenterol Hepatol 2020; 17:184-92.

\section{NICE 2019}

. Ulcerative colitis - Evidence reviews for induction of remission in mild-to-moderate ulcerative colitis. NICE guidelines [NG130]. Available at: https://www.nice.org.uk/guidance/ng130/ evidence/induction-of-remission-in-mildtomoderate-ulcerativecolitis-pdf-6777260893 (Accessed: 30/10/2019). 


\section{Norton 2017}

Norton C, Czuber-Dochan W, Artom M, Sweeney L, Hart A. Systematic review: interventions for abdominal pain management in inflammatory bowel disease. Aliment Pharmacol Ther 2017; 46:115-25.

\section{Ordas 2012}

Ordás I, Eckmann L, Talamini M, Baumgart DC, and Sandborn WJ. Ulcerative colitis. The Lancet 2012; 380(9853):1606-19. [DOI: https://doi.org/10.1016/ S0140-6736(12)60150-0]

\section{Pauly 2017}

Pauly NJ, Michailidis L, Kindred MG, Flomenhoft D, Lofwall MR, . Predictors of Chronic Opioid Use in Newly Diagnosed Crohn's Disease. Inflammatory Bowel Disease 2017; 23(6):1004-10. [DOI: 10.1097/MIB.0000000000001087]

\section{RevMan 2014 [Computer program]}

The Cochrane Collaboration Review Manager (RevMan). Version Version 5.3. Copenhagen: The Nordic Cochrane Centre: The Cochrane Collaboration, 2014.

\section{Srinath 2012}

Srinath AI, Walter C, Newara MC, Szigethy EM. Pain management in patients with inflammatory bowel disease: insights for the clinician. Therap Adv Gastroenterol 2012; 5(5):339-57. [DOI: $10.1177 / 1756283 \times 12446158]$

\section{Timmer 2011}

Timmer A, Preiss JC, Motschall E, Rücker G, Jantschek G, Moser G. Psychological interventions for treatment of inflammatory bowel disease. Cochrane Database of Systematic Reviews 2011, Issue 2. [DOI: 10.1002/14651858.CD006913.pub2]

\section{Wilkinson 2007}

Wilkinson $\mathrm{J}$ and Faleiro R. Acupuncture in pain management. BJA Education 2007; 7(4):135-8. [DOI: 10.1093/bjaceaccp/ mkm021\%J BJA Education]

\section{A P P E N D I CES}

\section{Appendix 1. Search strategies}

\section{Cochrane CCTR, CDSR search strategy (via Ovid)}

1. exp Pain/

2. pain $^{\star} . t w, k w$.

3. ((abdominal or abdomen) and (discomfort* or ache ${ }^{\star}$ or aching or migraine ${ }^{\star}$ or fibromyalgia* or neuralgia* or colic $\left.\left.{ }^{\star}\right)\right) . t w, k w$.

4. or/1-3

5. exp Colitis, Ulcerative/

6. Inflammatory Bowel Diseases/

7. (ulcer* adj5 colitis).tw,kw.

8. inflammatory bowel disease ${ }^{\star} . \mathrm{tw}, \mathrm{kw}$.

9. (colitis gravis or proctocolitis or procto colitis or mucosal colitis or colorectitis or UC).tw,kw.

10.or/5-9

11.4 and 10

\section{MEDLINE search strategy (via Ovid)}

1. exp Pain/

2. pain*.tw,kw.

3. ((abdominal or abdomen) and (discomfort* or ache* or aching or migraine* or fibromyalgia* or neuralgia* or colic $\left.\left.{ }^{\star}\right)\right) . t w, k w$.

4. or/1-3

5. exp Colitis, Ulcerative/

6. Inflammatory Bowel Diseases/

7. (ulcer* adj5 colitis).tw,kw.

8. inflammatory bowel disease ${ }^{\star} . \mathrm{tw}, \mathrm{kw}$.

9. (colitis gravis or proctocolitis or procto colitis or mucosal colitis or colorectitis or UC).tw,kw.

10.or/5-9

11.4 and 10

12.randomized controlled trial.pt.

13. controlled clinical trial.pt.

14.randomi?ed.ab.

15.placebo.ab.

16.drug therapy.fs. 
17.randomly.ab.

18.trial.ab.

19.groups.ab.

20.or/12-19

21.exp animals/ not humans/

22.20 not 21

23.11 and 22

24.cochrane database of systematic reviews.jn. or search ${ }^{\star}$.tw. or meta analysis.pt. or medline.tw. or systematic review.tw.

25.11 and 24

26.23 or 25

(lines 12-22: [Cochrane Handbook RCT filter - sensitivity max version]

line 24:[Wong 2006 - systematic reviews filter - specificity maximizing version])

\section{PsycINFO search strategy (via Ovid)}

1. exp Pain/

2. Pain Measurement/

3. Pain Perception/

4. Pain Management/

5. pain*.tw.

6. ((abdominal or abdomen) and (discomfort* or ache* or aching or migraine ${ }^{\star}$ or fibromyalgia* or neuralgia* or colic $\left.\left.{ }^{\star}\right)\right)$. tw.

7. or/1-6

8. exp Ulcerative Colitis/

9. (ulcer* adj5 colitis).tw.

10.inflammatory bowel disease*.tw.

11. (colitis gravis or proctocolitis or procto colitis or mucosal colitis or colorectitis or UC).tw.

12.or/8-11

13.7 and 12

14. (control* or random*).tw. or exp Treatment/

15.13 and 14

\section{CINAHL search strategy (EBSCO)}

S1. MH "Pain+"

S2.TI pain* OR AB pain*

S3. TI ( (abdominal or abdomen) and (discomfort* or ache* or aching or migraine* or fibromyalgia* or neuralgia* or colic ${ }^{\star}$ ) ) OR AB ( (abdominal or abdomen) and (discomfort ${ }^{\star}$ or ache* or aching or migraine* or fibromyalgia* or neuralgia* or colic $\left.^{\star}\right)$ )

S4. S1 OR S2 OR S3

S5. (MH "Colitis, Ulcerative")

S6. TI ( ulcer ${ }^{\star}$ and colitis ) OR AB ( ulcer* and colitis )

S7. TI inflammatory bowel disease* OR AB inflammatory bowel disease ${ }^{\star}$

S8. TI ( colitis gravis or proctocolitis or procto colitis or mucosal colitis or colorectitis or UC) OR AB ( colitis gravis or proctocolitis or procto colitis or mucosal colitis or colorectitis or UC)

S9. S5 OR S6 OR S7 OR S8

S10. S4 AND S9

S11. MH "prognosis+" OR MH "study design+" OR random*

S12. S10 AND S11

(Line S11: [Wong 2006 "CINAHL therapy studies" filter - best sensitivity version])

\section{AMED search strategy (via Ovid)}

1. pain*.tw.

2. ((abdominal or abdomen) and (discomfort* or ache* or aching or migraine* or fibromyalgia* or neuralgia* or colic $\left.\left.{ }^{\star}\right)\right)$.tw.

3. $\mathrm{or} / 1-2$

4. (ulcer* adj5 colitis).tw.

5. inflammatory bowel disease ${ }^{\star} . t w$. 
6. (colitis gravis or proctocolitis or procto colitis or mucosal colitis or colorectitis or UC).tw.

7. or $/ 4-6$

8. 3 and 7

\section{ClinicalTrials.gov search strategy (Adavnced Search)}

Condition/ Disease: (ulcerative colitis OR inflammatory bowel disease OR colitis gravis OR proctocolitis OR procto colitis OR mucosal colitis OR colorectitis)

Other terms: (pain OR pains OR ache* OR aching OR fibromyalgia* OR neuralgia* OR colic*)

Study Type: Interventional Studies

\section{WHO ICTRP Search Portal search strategy (Standard search)}

pain* AND ulcerative colitis OR

ache $^{\star}$ AND ulcerative colitis OR

aching AND ulcerative colitis OR

colic* AND ulcerative colitis OR

pain* AND inflammatory bowel disease OR

ache* AND inflammatory bowel disease OR

aching AND inflammatory bowel disease OR

colic* AND inflammatory bowel disease OR

pain* AND proctocolitis OR

ache* AND proctocolitis OR

aching AND proctocolitis OR

colic* AND proctocolitis OR

pain* AND colorectitis OR

ache* AND colorectitis OR

aching AND colorectitis OR

colic ${ }^{\star}$ AND colorectitis

\section{H I S T O R Y}

Protocol first published: Issue 4, 2020

\section{CONTRIBUTIONS OFAUTHORS}

Zipporah Iheozor-Ejiofor: developed the protocol; coordinated the protocol development; produced the first draft of the protocol; contributed to writing or editing the protocol; made an intellectual contribution to the protocol; approved the final version of the protocol prior to submission; and is a guarantor of the protocol

Morris Gordon: conceived the review question; developed the protocol; secured funding; contributed to writing or editing the protocol; made an intellectual contribution to the protocol; advised on the protocol; approved the final version of the protocol prior to submission; and is a guarantor of the protocol.

Anthony K Akobeng: developed the protocol; made an intellectual contribution to the protocol; advised on the protocol; approved the final version of the protocol prior to submission.

\section{DECLARATIONS OF INTEREST}

Zipporah Iheozor-Ejiofor: None known

Morris Gordon: Since August 2016, I have received travel fees to attend international scientific and training meetings from Pharma companies. These grants included no honoraria, inducement, advisory role or any other relationship and were restricted to the travel and meeting related costs of attending such meetings. These include: DDW May 2017, World Congress of Gastroenterology October 2017, DDW May 2018, Advances in IBD December 2018, DDW May 2019.

The companies include: Biogaia (2017-19), Ferring (2018), Allergan (2017), synergy (bankrupt - 2018) and Tillots (2017-19).None of these companies have had any involvement in any works completed by me and I have never had any payments for any other activities for them, as confirmed below. From these date onwards, I have made a personal undertaking to take no further funds from any pharmaceutical or formula company in any form for travel or other related activities. This is to lift the limitations such funding has on my ability to act as a first and corresponding author on reviews, in line with the Cochrane policies on such matters and is reported in line with these policies. These current declarations will expire over the next 3 years and this statement updated regularly to reflect this.

Anthony K Akobeng: None known. 